\title{
Abstract
}

\section{Evaluation of the Dengue Surveillance System in Khyber Pakhtunkhwa Province, Pakistan, in 2020}

\author{
Omar Sharif Khan, MPH, MBBS \\ Health Department Khyber Pakhtunkhwa, Peshawar, Pakistan
}

\author{
Corresponding Author: \\ Omar Sharif Khan, MPH, MBBS \\ Health Department Khyber Pakhtunkhwa \\ House no.87, street 4, sector H2, Phase 2, Hayatabad \\ Peshawar, 25000 \\ Pakistan \\ Phone: 923052223335 \\ Email: dromarsharifkhan@gmail.com
}

\section{Abstract}

Background: Installation and actualization of a disease surveillance system are prerequisites for early detection of outbreaks. Prompt response is possible when a robust surveillance system is in place. Dengue is one of the many diseases endemic to Pakistan and is potentially fatal.

Objective: This study aimed to assess the current dengue surveillance system and its performance and to provide recommendations to stakeholders for its actualization and improvement.

Methods: A cross-sectional study was conducted in 2020 to document the outcomes. The evaluation was guided by the updated Centers for Disease Control and Prevention guidelines for public health surveillance for the year 2019. A structured questionnaire was designed and piloted to estimate the simplicity, flexibility, acceptability, and stability of the current dengue surveillance system. The sample included 45 provincial- and district-level staff involved in dengue surveillance. Provincial data on dengue were analyzed to evaluate completeness, quality, positive predictive value, sensitivity, and representativeness. Field visits to districts were performed to assess data flow and timeliness.

Results: The reporting rate ranged from $12 / 100,000$ in 2017 to $21 / 100,000$ in 2019 , with a total of 7641 reported cases in the province. The mean time of reporting cases was 1 day (range 0-2 days). The simplicity of the dengue surveillance system was at $90 \%$ with respect to structure and data flow. The stability of the system was at $84 \%$ because of data backup. System flexibility was at $81 \%$ and allowed the addition and modification of variables. The average completeness of the selected variables was $65 \%$. About $59 \%$ of the staff interviewed considered the system acceptable. Data quality was suboptimal at $48 \%$. The representativeness of the system was at $40 \%$, and it was mainly representative of secondary and tertiary health care hospitals, particularly inpatients. The system positive predictive value for dengue was $15 \%$ and sensitivity was $14 \%$, which were below par. The dengue surveillance system can detect dengue outbreaks early.

Conclusions: An immediate, collaborative, multisectoral, and transdisciplinary plan is needed to enhance reporting from all health facilities. Adequate government funding is needed to improve data quality, and a monitoring mechanism is needed at all levels for prompt functioning of the surveillance system.

(iproc 2022;8(1):e36584) doi: $10.2196 / 36584$

\section{KEYWORDS}

evaluation; dengue; surveillance; Khyber Pakhtunkhwa 
Edited by Y Khader; this is a non-peer-reviewed article. Submitted 18.01.22; accepted 24.01.22; published 08.02.22.

Please cite as:

Khan OS

Evaluation of the Dengue Surveillance System in Khyber Pakhtunkhwa Province, Pakistan, in 2020

iproc 2022;8(1):e36584

URL: https://www.iproc.org/2022/1/e36584

doi: $10.2196 / 36584$

PMID:

(C) Omar Sharif Khan. Originally published in Iproceedings (https://www.iproc.org), 08.02.2022. This is an open-access article distributed under the terms of the Creative Commons Attribution License (https://creativecommons.org/licenses/by/4.0/), which permits unrestricted use, distribution, and reproduction in any medium, provided the original work, first published in Iproceedings, is properly cited. The complete bibliographic information, a link to the original publication on https://www.iproc.org/, as well as this copyright and license information must be included. 